\title{
Superradiance or total reflection?
}

\author{
András László ${ }^{1,2}$ István Rácz ${ }^{2}$ \\ ${ }^{1}$ CERN, CH-1211 Genéve 23, Switzerland \\ ${ }^{2}$ Wigner RCP, H-1121 Budapest,Hungary \\ E-mail: laszlo.andras@wigner.mta.hu, \\ racz.istvan@wigner.mta.hu
}

\begin{abstract}
Numerical evolution of massless scalar fields on Kerr background is studied. The initial data specifications are chosen to have compact support separated from the ergoregion and to yield nearly monochromatic incident wave packets. The initial data is also tuned to maximize the effect of superradiance. Evidences are shown indicating that instead of the anticipated energy extraction from black hole the incident radiation fail to reach the ergoregion rather it suffers a nearly perfect reflection.
\end{abstract}

\section{Introduction}

To motivate our investigations let us mention first that the stability of the Kerr family of black hole solutions within the space of the vacuum solutions to the Einstein equations is one of the most important unresolved issues in general relativity. The ultimate goal is to provide boundedness and decay statements for solutions of the vacuum Einstein equations around the members of the Kerr family.

It may be a surprise that - even nowadays when numerical simulations of binary black hole systems becomes a daily routine essentially all work concerning the aforementioned black hole stability problem has been confined to the linearized setting. Indeed, considerations are restricted to study the solutions to the Klein-Gordon equation

$$
\square_{K} \Phi=0
$$

on Kerr background. This is done with the hope that the understanding of these simplified scalar perturbations are good preparations to the 
study of more complicated problem of full but yet linear gravitational perturbations.

It should also be mentioned that all the available analytic proofs justifying the linear stability for scalar perturbations, even in this simplest possible setting, are known to be restricted to the case of slowly rotating subextremal Kerr black holes [1, 2].

\section{Superradiance}

To start off recall first that superradiance was discovered in the early 70 's as a new phenomenon and it may be associated with the names of Misner, Zel'dovich and Starobinskii [3, 4, 5. It is also considered to be the wave analog of the Penrose process and it is supposed to allow energy to be extracted from black holes.

The common belief related to the interaction of black holes with incident radiation is summarized as "...if scalar, electromagnetic or gravitational wave is incident upon a black hole, part of the wave (the "transmitted wave") will be absorbed by the black hole and part of the wave ( the "reflected wave") will escape to infinity" [6]. Recall that by using the Teukolsky equation [7] the evolution of scalar, electromagnetic and gravitational perturbations can be investigated within the same setting. It is also important to be mentioned that all the conventional arguments ending up with superradiance, including the ones based on Teukolsky's equation, refer to properties of individual modes [8].

Interestingly, as it was pointed out first by Bekenstein [9], whenever superradiance happens it can be seen to be completely consistent with the laws of black hole thermodynamics. It is also worth to be mentioned some of the expectations relevant for scalar perturbations. It was claimed in 10 "Starobinskii made an asymptotic expansion for the reflection coefficient and found a relative gain of energy of about $5 \%$ for $m=1$ and less than $1 \%$ for $m \geq 2$ ".

\section{Superradiance in mode analysis}

It was realized first by Carter 11 that the temporal Fourier transform, $\mathscr{F} \Phi=\frac{1}{\sqrt{2 \pi}} \int_{-\infty}^{+\infty} \Phi e^{i \omega t} d t$, of a solution to 11 may be decomposed as

$$
\mathscr{F} \Phi\left(\omega, r_{*}, \vartheta, \varphi\right)=\frac{1}{\sqrt{r^{2}+a^{2}}} \sum_{\ell=0}^{\infty} \sum_{m=-\ell}^{\ell} R_{\ell, \omega}^{m}\left(r_{*}\right) S_{\ell, a \omega}^{m}(\vartheta, \varphi),
$$

where $t, r_{*}, \vartheta, \varphi$ are local coordinates, while $\omega$ is the frequency in the time translation direction. In (2) $S_{\ell, a \omega}^{m}$ denotes the oblate spheroidal harmonic functions, with oblateness parameter $a \omega$, and with angular momentum 
quantum numbers $\ell, m$. The functions $S_{\ell, a \omega}^{m}$ are eigenfunctions of a selfadjoint operator.

For the radial functions $R_{\ell, \omega}^{m}$ in 2 a one-dimensional Schrödinger equation of the form

$$
\frac{d^{2} R_{\ell, \omega}^{m}}{d r_{*}^{2}}+\left[\left(\omega-\frac{m a}{r^{2}+a^{2}}\right)^{2}+\left(r-r_{H}\right) \cdot V_{\ell, \omega}^{m}\left(r_{*}\right)\right] R_{\ell, \omega}^{m}=0,
$$

can be derived from (1), with suitable real potentials $V_{\ell, \omega}^{m}\left(r_{*}\right)$.

The "physical solutions" to (3) are supposed to possess the asymptotic behavior

$$
R_{\ell, \omega}^{m} \sim \begin{cases}e^{-i \omega r_{*}}+\mathcal{R} e^{+i \omega r_{*}} & \text { as } r \rightarrow \infty \\ \mathcal{T} e^{-i\left(\omega-m \Omega_{H}\right) r_{*}} & \text { as } r \rightarrow r_{H},\end{cases}
$$

where $\Omega_{H}$ stands for the angular velocity of the black hole with respect to the asymptotically stationary observers, while $\mathcal{R}$ and $\mathcal{T}$ denotes the reflection and transmission coefficients. Notice that these boundary conditions presumes the existence of a transmitted wave submerging into the ergoregion.

By evaluating the Wronskian of the associated fundamental solutions, "close" to infinity and "close" to the horizon, it can be shown that

$$
\left(\omega-m \Omega_{H}\right)|\mathcal{T}|^{2}=\left(1-|\mathcal{R}|^{2}\right) \omega .
$$

In virtue of (5) it follows that whenever $|\mathcal{R}|>1$-or equivalently whenever $|\mathcal{T}|$ does not vanish and the inequality

$$
0<\omega<m \Omega_{H}
$$

holds - positive energy is supposed to be acquired by the backscattered scalar mode due to its interaction with the Kerr black hole.

\section{Numerical studies of superradiance}

So far our considerations have been restricted to the study of individual modes. However the investigation of the linear stability problem [12, 13, 1, 2 taught us the lesson that statements which are valid at the level of individual modes typically do not imply statements for finite energy solutions composed by infinitely many modes.

This section is to reveal some of our pertinent numerical results. Before proceeding let us mention that the first time domain studies of superradiance was carried out long time ago [14, 15. The scale of energy extraction was found to be smaller than the estimates recalled above. The numerical results reported below were derived by making use of our code called GridRipper which is a fully spectral in the angular directions while the dynamics in the complementary $1+1$ Lorentzian spacetime is followed by making use of a fourth order finite differencing scheme [16, 17] (see also [18]). 


\subsection{The initial data}

To have an incident scalar wave - to study the way a to be superradiant solution acquires extra energy by submerging into the ergoregion-, in a sufficiently small neighborhood of the initial data surface in the asymptotic region, the solution was assumed to posses the form

$$
\Phi\left(t, r_{*}, \vartheta, \tilde{\varphi}\right) \approx e^{-i \omega_{0}\left(r_{*}-r_{* 0}+t\right)} f\left(r_{*}-r_{* 0}+t\right) Y_{\ell}^{m}(\vartheta, \tilde{\varphi}) .
$$

where $f: \mathbb{R} \rightarrow \mathbb{C}$ is a smooth function of compact support and $\omega_{0}, r_{* 0}$ are real parameters. This suggest the use of initial data

$$
\begin{aligned}
\phi\left(r_{*}, \vartheta, \tilde{\varphi}\right) & =e^{-\mathrm{i} \omega_{0}\left(r_{*}-r_{* 0}\right)} f\left(r_{*}-r_{* 0}\right) Y_{\ell}^{m}(\vartheta, \tilde{\varphi}), \\
\phi_{t}\left(r_{*}, \vartheta, \tilde{\varphi}\right) & =-\mathrm{i} \omega_{0} \phi\left(r_{*}, \vartheta, \tilde{\varphi}\right)+e^{-\mathrm{i} \omega_{0}\left(r_{*}-r_{* 0}\right)} f^{\prime}\left(r_{*}-r_{* 0}\right) Y_{\ell}^{m}(\vartheta, \tilde{\varphi}),
\end{aligned}
$$

where $f^{\prime}$ denotes the first derivative of $f: \mathbb{R} \rightarrow \mathbb{C}$. The Fourier transform, $\mathscr{F} \Phi$, of the approximate solution (7) reads as

$$
\mathscr{F} \Phi\left(\omega, r_{*}, \vartheta, \tilde{\varphi}\right) \approx e^{-\mathrm{i} \omega\left(r_{*}-r_{* 0}\right)} \mathscr{F} f\left(\omega-\omega_{0}\right) Y_{\ell}^{m}(\vartheta, \tilde{\varphi}),
$$

where $\omega$ is the temporal frequency and $\mathscr{F} f$ stands for the Fourier-transform of $f$. Notice that $\mathscr{F} f$ plays the role of a frequency profile, which guaranties that whenever $\mathscr{F} f$ is chosen to be a sufficiently narrow the approximate solution (7) has to be close to a monochromatic wave packet, which for suitable value of $\omega_{0}$ becomes to be superradiant (for more details see [17]).

\subsection{Numerical results}

The plots shown below refer to evolution of a pure quadrupole type initial data with radial profile function $f: \mathbb{R} \rightarrow \mathbb{C}$

$$
f_{w}(x)=\left\{\begin{aligned}
e^{\left[-\left|\frac{w}{x+\frac{w}{2}}\right|-\left|\frac{w}{x-\frac{w}{2}}\right|+4\right]}, & \text { if } x \in\left[-\frac{w}{2}, \frac{w}{2}\right] \\
0, & \text { otherwise }
\end{aligned}\right.
$$

which is a smooth function of the real variable $x$ with compact support $\left[-\frac{w}{2}, \frac{w}{2}\right]$, and with initial parameters $M=1, a=0.99, \ell=m=2$, $\omega_{0}=\frac{1}{2} m \Omega_{H}, r_{* 0}=31.823$.

It is important to be sure (see Fig 1 ) that the above choice yield a solution with the expected frequency profile.

The time dependence of the radial energy and angular momentum distributions, along with the complete power spectrum, are shown ${ }^{1}$ on the succeeding figures, Fig 2 and 3 . These figures indicate that the reported nearly perfect reflection do really happen for the considered to be superradiant solution. 


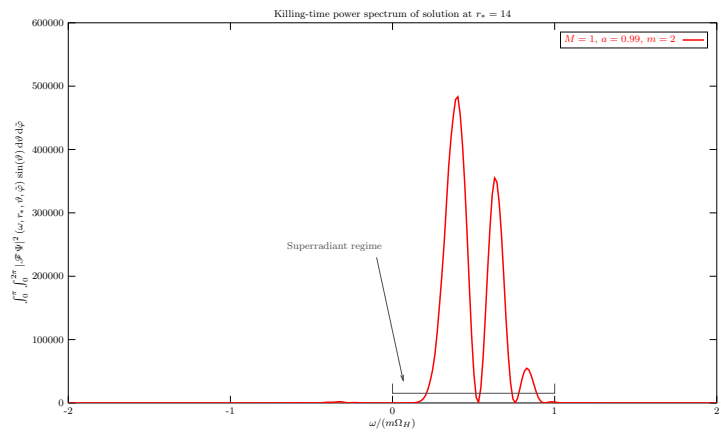

Figure 1. The power spectrum of the to be superradiant solution at $r_{*}=14$, located between the compact support and the black hole.

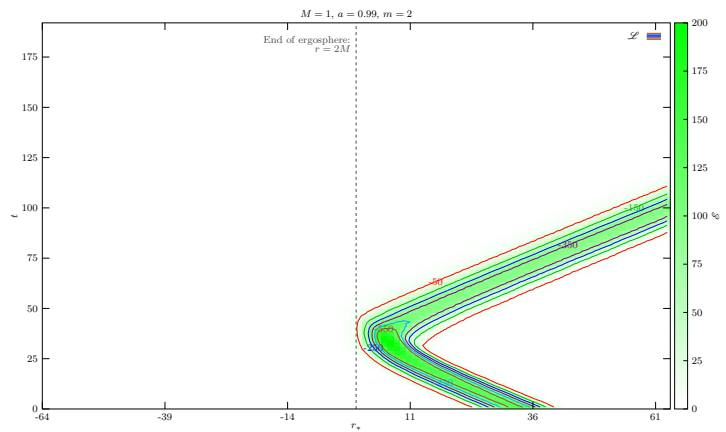

Figure 2. The radial energy and angular momentum distributions.

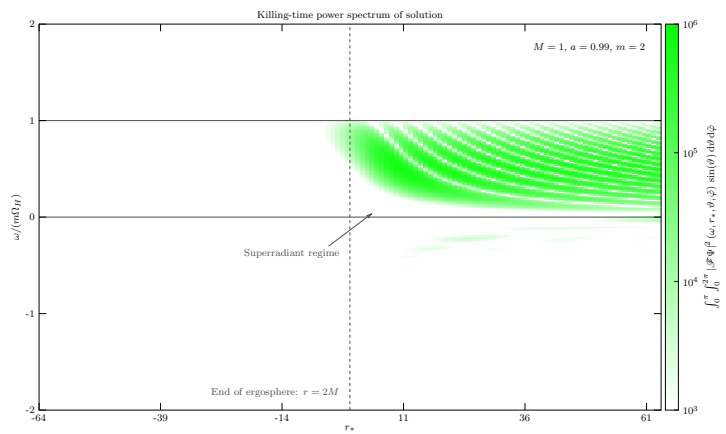

Figure 3. The radial distribution of the power spectrum. 


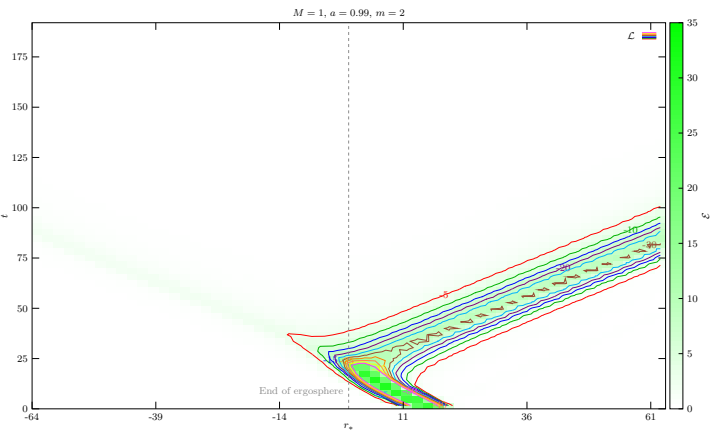

Figure 4. The frequency $\omega_{0}$ of this nearly superradiant solution is the same as before. Only the support is shifted to get a submerging part.

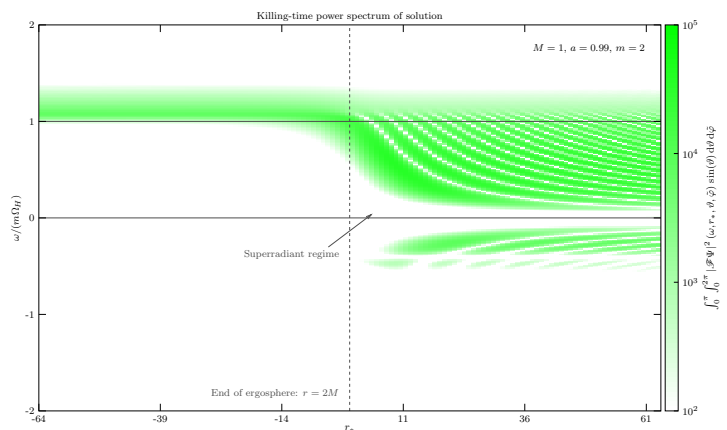

Figure 5. The untuned solution has a submerging part but its power spectrum jumps out of the superradiant domain on reaching the ergoregion.

It is also informative to have a look at the corresponding figures (see Fig 4 and 5 for an almost to be superradiant solution yielded by shifting the compact support towards the black hole - decreasing slightly thus the angular momentum of the radiation with respect to its energy - while all the other parameters remained intact. Notice that, in virtue of Fig 5 , the frequency content of the part of the incident wave packet, submerging into the ergoregion, gets completely evacuated from the superradiant domain.

It is also important to be mentioned that according to the accuracy of our code no energy extraction - or, at least, not more than $10^{-3}$ times of

1 Note that on all the included 2-dimensional plots the indicated quantities are integrated with respect to the radial degrees of freedom. 


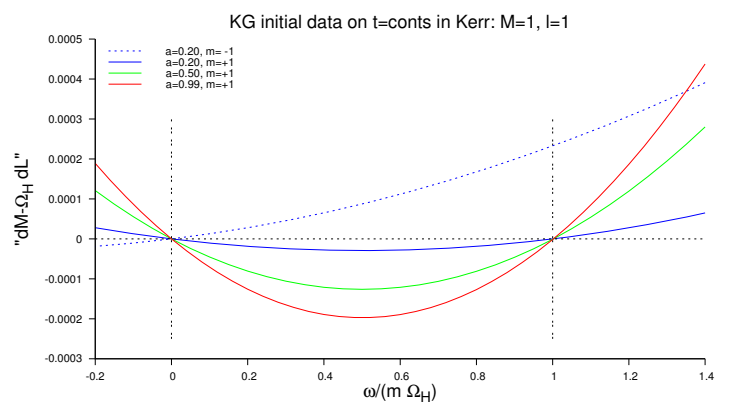

Figure 6. The energy and angular momentum content of initial data specifications are compared. For to be superradiant configurations the incident wave cannot deliver its full energy and angular momentum to the black hole unless the second law of black hole thermodynamics is violated.

the initial energy — happened in either of these (or analogous) simulations.

\section{Summary}

The numerical evolution of massless Klein-Gordon field on Kerr background, arising from initial data with compact support in the asymptotic region, was considered. The incident wave packet was tuned to maximize the effect of superradiance.

For perfectly tuned initial data no energy extraction could be observed. Significant part of the incident radiation fail to reach the ergoregion and the time evolution mimics the phenomenon of a total reflection.

To get some insight about the physical mechanism beyond the nearly total reflection it turned out to be useful to compare the energy and angular momentum content of initial data specifications. Fig 6 is to demonstrate that far too much angular momentum is stored by the to be superradiant wave packets, as $d E<\Omega_{H} d L$ holds for them, which-in virtue of the second law of black hole thermodynamics - does not allow these wave packets to enter the black hole region. Accordingly, the observed nearly total reflection may be considered as the field theoretical analog of the phenomenon in Wald's thought experiments [19] demonstrating, in the early 70', that a Kerr black hole does not capture a particle that would cause a violation of the relation $m^{2} \geq a^{2}+e^{2}$.

\section{Acknowledgments}

This research was supported in part by OTKA grant K67942. 


\section{References}

[1] Andersson, L. and Blue, P., "Hidden symmetries and decay for the wave equation on the Kerr spacetime", arXiv:0908.2265v2 (2009)

[2] Dafermos, M. and Rodnianski, M., "Decay for solutions of the wave equation on Kerr exterior spacetimes I-II: The cases $|a| \ll M$ or axisymmetry", arXiv:1010.5132v1 (2010)

[3] Misner, C.W., "Interpretation of Gravitational-Wave Observations", Phys. Rev. Lett. 28, 994 (1972)

[4] Zel'dovich Ya.B., Zh. Eksp. Teor. Fiz. 62, 2076 (1971)

[5] Starobinskii, A.A., "Amplification of waves during reflection from rotating black hole", Zh. Eksp. Teor. Fiz. 64, 48 (1973)

[6] Wald, R.M., General relativity, University of Chicago Press, Chicago (1984)

[7] Teukolsky, S.A., "Rotating Black Holes: Separable Wave Equations for Gravitational and Electromagnetic Perturbations" Phys. Rev. Lett. 29, 1114 (1972)

[8] Press, W.H. and Teukolsky, S.A., "Perturbations of a Rotating Black Hole. II. Dynamical Stability of the Kerr Metric", Astrophys. J. 185, 649 (1973)

[9] Bekenstein, J., "Extraction of Energy and Charge from a Black Hole", Phys. Rev. D 7, 949 (1973)

[10] Finster, F., Kamran, N., Smoller. J. and Yau, S.T., "A rigorous treatment of energy extraction from rotating black hole", Commun. Math. Phys. 287829 (2009)

[11] Carter, B., "Hamilton-Jacobi and Schrödinger separable solutions of Einstein's equations", Commun. Math. Phys. 10, 280 (1968)

[12] Wald, R.M., "Note on the stability of the schwarzschild metric", J. Math. Phys. 20, 1056 ?1058 (1979)

[13] Kay, B.S. and Wald, R.M.,"Linear stability of Schwarzschild under perturbations which are nonvanishing on the bifurcation two sphere", Class. Quant. Grav., 4, 893 (1987)

[14] Krivan, W., Laguna, P., Papadopoulos, P. and Andersson, N., "Dynamics of perturbations of rotating black holes", Phys. Rev. D 56, 3395 (1997)

[15] Andersson, N., Laguna, P., Papadopoulos, P., "Dynamics of Perturbations of Rotating Black Holes. II. A note on superradiance", Phys. Rev. D 58, 087503 (1998)

[16] The GridRipper 3+1d PDE solver: http://www.rmki.kfki.hu/ gridripper

[17] Csizmadia, P., László, A. and Rácz, I., "On the Use of Multipole Expansion in Time Evolution of Non-linear Dynamical Systems and Some Surprises Related to Superradiance", arXiv:1207.5837 (2012)

[18] Csizmadia, P., László, A. and Rácz, I., "Linear waves on fixed Kerr background and their relevance in jet formation", Journ. Phys. Conf. Ser. 218, 012007 (2010)

[19] Wald, R.M., Gedanken experiments to destroy a black hole", Annals of Physics 83, 548 (1974) 